\title{
Development and Feasibility Testing of a Physical Activity Intervention For Youth With Anxiety and Depression: a Study Protocol
}

\section{Arne Kodal ( $\square$ arne.kodal@helse-bergen.no)}

Haukeland University Hospital: Haukeland Universitetssjukehus

John J. Reilly

University of Strathclyde

Fiona Muirhead

Strathmore University

Gro Janne $H$. Wergeland

Haukeland University Hospital: Haukeland Universitetssjukehus

Paul Joachim Bloch Thorsen

Haukeland University Hospital: Haukeland Universitetssjukehus

Lars Peder Bovim

Hogskulen pa Vestlandet

Irene Bircow Elgen

Bergen University: Universitetet i Bergen

\section{Study Protocol}

Keywords: Anxiety, depression, youth, physical activity, Intervention

Posted Date: October 1st, 2021

DOl: https://doi.org/10.21203/rs.3.rs-917447/v1

License: (c) (i) This work is licensed under a Creative Commons Attribution 4.0 International License.

Read Full License

Version of Record: A version of this preprint was published at Pilot and Feasibility Studies on March 2nd, 2022. See the published version at https://doi.org/10.1186/s40814-022-01010-6. 


\section{Abstract}

Background: Anxiety and depressive disorders in children and adolescents are highly prevalent and account for more than half of all youth psychiatric disorders. Left untreated, anxiety and depression leads to numerous detrimental outcomes, including reduced quality of life, psychiatric and somatic comorbidity and even reduced lifespan. This puts a large strain on child and adolescent mental healthcare services (CAMHS) to provide effective treatments. However, even when provided the best evidence based treatment, between 40-50 \% continue to report significant symptom burdens. Thus, there is an immediate need for supplemental and/or new treatment approaches. Physical activity as a supplementary treatment may be such an approach. However, research investigating this approach within this population is scant. This protocol paper describes the development and feasibility trial of a physical activity based intervention targeting anxiety and depressive symptoms in youth treated in CAMHS.

Methods/design: The study is based on the UK Medical Council Research Framework (MRC) for developing and evaluating complex interventions. Feasibility and acceptability of the physical activity intervention (Confident, Active and Happy Youth) will be evaluated in an uncontrolled open-label trial using qualitative and quantitative data. Twenty youths with anxiety and/or depressive symptoms will be recruited. Acceptability of assessment procedures, the intervention, and perceived benefits and barriers to participation will be assessed, and qualitative interviews with participants, caregivers and referring specialists will explore contextual and practical factors associated with intervention delivery. Physical activity will be measured using the Actigraph GT3X+ monitor at baseline and post-intervention and change in anxiety and depression will be assessed.

Discussion: This study will contribute to the development of supplementary physical treatment interventions for youth with anxiety and depression in contact with CAMHS. The goal is to examine new avenues of treatment that ultimately may improve upon current treatment outcomes of anxiety and depression. This work will be in preparation for a future definitive RCT of this approach, in line with the MRC framework.

\section{Trial registration:}

ClnicalTrials.gov, NCT05049759. Registered 19 August 2021 - Retrospectively registered.

\section{Background}

Internalizing disorders (i.e., anxiety and depressive disorders), represent as a group the most common mental health disorders among children and adolescents (hereafter youth), with a prevalence of $6.5 \%(\mathrm{Cl}$ 95\% 4.7-9.1) and $2.6 \%(\mathrm{Cl} 95 \% 1.7-3.9)$ respectively $(1,2)$. These disorders tend to develop early in life, and short- and long-term consequences include reduced quality of life, psychiatric and somatic comorbidity, disability, loss of education and/or work, suicide and reduced life-span $(3,4)$. According to the World Health Organisation, anxiety and depressive disorders are major health problems with large- 
scale societal and economic consequences (WHO, 2017). Anxiety and depression are among the top five causes of overall disease burden among youth in Europe (5).

Discouragingly however, even when provided the best evidence based treatment, post-treatment remission rates for anxiety and depressive disorders in youth is just slightly above chance e.g. $50 \%$, (mixed anxiety: $50.7 \% \mathrm{Cl}$ : 45.3-56.2; depression: 53.2\%, Cl: 34.6-70.9 (6). Apart for the consequences for the individual, such suboptimal treatment outcomes also place a large strain on treatment services, which are dependent upon sufficient capacity and effective treatment. If patients do not recover or experience disorder relapse following treatment, these patients require further treatment, thus excluding new patients from gaining access to treatment. In light of the prevalence of these disorders, short- and long-term consequences and the suboptimal effect of current treatments, there is a pressing need for development of new and supplementary interventions to improve recovery rates (6-8).

In recent years physical activity has gained traction as a promising area in effective mental health treatments $(9,10)$, and physical activity is identified as a key modifiable factor in people with mental illness (11). Meta-analyses of the effects of physical activity in the treatment of anxiety and depression in adult populations indicate small to moderate effects (12-14). This knowledge is now implemented in adult depression treatment recommendations such as those provided by The European Psychiatric Association (15). Unfortunately, the amount of research on the topic in paediatrics is substantially less (9, 16). However, new evidence is starting to accumulate. Three recent meta-analyses investigating the effect of physical activity on youth depression find small to moderate effects (16-18). Studies examining the effects of physical activity on anxiety disorders in youth are even fewer with only one meta-analysis. In this meta-analysis, Carter et al. (2021) conclude that physical activity is potentially effective in reducing anxiety symptoms within non-clinical populations of youth, while evidence within clinical populations is insufficient to draw any clear conclusions. All the cited meta-analyses call for well-designed research with youth populations - particularly clinical youth populations (16-19).

The ingredients by which physical activity interventions impacts anxiety and depression are complex and multifaceted (20). Current available research are either too limited or demonstrate mixed results hampering attempts to tease out specific intervention characteristics required. However, the cited metaanalyses do point to a few common ingredients across the studies associated with positive effect. These ingredients included supervised, aerobic- and group-based activity of moderate-to-vigorous intensity, with participants engaging in activities multiple times per week over a time period of at least six to eight weeks (16-19). Questions concerning optimum dosage, type of activity and energy expenditure are still unclear.

Focusing on the more specific mechanisms of effect of physical activity on anxiety and depression in youth, several biological, psychosocial and behavioural factors are likely involved. Neurobiological mechanisms may include processes that are both disrupted or dysregulated and potentially modulated by physical activity. Processes may include inflammatory and oxidative stress responses, neurogenesis, modulation of monoamines (e.g., serotonin), and HPA axis regulation $(21,22)$. Hillman et al., found that a program targeting cardiorespiratory fitness resulted in increased neural underpinnings of attentional 
resources on tasks requiring improved inhibition and cognitive flexibility (23). Deficits in inhibition and cognitive control are strongly associated with anxiety and depression in youth (24).

In regards to psychosocial processes, physical activity can increase opportunities for social interaction, social connectedness, experiences of mastery and lead to increased self-efficacy and independence (20, 25). Problems and deficits within these areas are closely associated with anxiety and depression in youth $(26,27)$. Exposure therapy is the treatment of choice for anxiety disorders in youth (6). Similarly, physical activity is also assumed to affect anxiety and depression through exposure towards an avoided object and/or situation, i.e. physical activity itself, induces bodily sensations and reactions that otherwise might be interpreted as symptoms of anxiety and/or be negatively appraised. Exposure to such sensations and reactions in this setting is thus assumed to be associated with non-threatening experiences, leading to normalization of these experiences and a more appropriate interpretation of these (Kandola et al., 2018).

In terms of behavioural mechanisms, physical activity may improve sleep, self-regulation and coping skills (20). The interplay of the described mechanisms above most likely also highly idiosyncratic to the individual, based on their prior beliefs and experiences of physical activity.

\section{Development of a supplementary intervention}

The development of a supplementary physical activity intervention took place in the Child and Adolescent Mental Health Services, Haukeland University Hospital, Bergen, Norway from 2018-2020. A work and management group consisting of clinicians and researchers, and a trial steering committee consisting of stakeholders, clinicians, users and parents identified key intervention components and a suitable delivery format. In addition, the group identified and outlined necessary organizational infrastructure, internal work procedures and team composition in order to deliver the treatments effectively.

The target goals of the program link closely to interrelated core symptoms of anxiety and depression; reduced or low levels of physical activity (28); lack of confidence in one's ability to cope with situations that incites distress and/or fear; decreased willingness to engage in and avoidance of situations that may incite distress and/or fear $(27,29)$. Finally, a core symptom particularly with depression is lowered mood. Taken together, the primary aim of the intervention is to alleviate these core symptoms and supplement ongoing treatment in CAMHS, which translates to helping youth become more confident, active and happy, hence the name of the intervention: Confident, Active and Happy Youth (CAH-Y).

The primary aims of the intervention are to:

- help youth become more confident, more happy and more physically active.

- Reduce symptoms of anxiety and depression.

Secondary aims of the $\mathrm{CAH}-\mathrm{Y}$ intervention are: 
- To motivate and support youths with anxiety/depression symptoms to initiate and maintain an active lifestyle and reduce sedentary behavior.

- To help foster integration of physical activity into current treatment approaches for internalizing disorders in youth in treatment in CAMHS.

While the effects of the physical activity itself are important and required in the intervention, there is also a need to address underlying reasons why youth with anxiety and depression avoid and/or have difficulty doing physical activity. To this end, the CAH-Y intervention draws on Self Determination Theory (25) and Inhibitory Learning Theory (30). Self Determination Theory provides a framework with which to understand participant experiences with physical activity and their motivation (as opposed to avoidance) towards physical activity. The framework focuses on ways to enhance learning and intrinsic motivation concerning both increased activity and behavior and symptom change (25). More specifically, the intervention attempts to satisfy youths' basic psychological needs for autonomy (e.g., providing youths with choice), competence (e.g., adapting exercises to meet the needs/fitness levels of students), and relatedness (e.g., promoting an inclusive group environment). Inhibitory learning theory provides a generic framework to understand the bi-directional interaction between cognition, affects and behavior in regards to anxiety and depression and in particular the role of (physical activity) avoidance as a coping and affect-regulation strategy, that both causes and maintains these disorders (30).

Drawing together these theories and known components, a manual was developed detailing a supervised, therapist led, group-based physical activity program involving aerobic exercise bi-weekly over a course of seven weeks. Further details on the intervention are detailed below in the intervention section. The full treatment manual (in Norwegian) is available on request by contacting the corresponding author.

In line with guidance from the UK Medical Council Research Framework (MRC) (31), the present study is an initial step in determining if the developed intervention is feasible and acceptable for youth with internalizing disorders in CAMHS. A mixed-methods approach was chosen to explore indications of participants' response and view to the intervention and thereby gain a more full understanding of the intervention acceptability (32). The present study also includes a calculation of effect size estimates for outcome measures to estimate the sample size of a future definitive randomized controlled trial (RCT).

The principal aims of this study are as follows:

1. Recruitment rate, willingness to participate, attendance, treatment retention and adherence

2. The appropriateness and practicality of the designed intervention in the proposed settings

3. The acceptability of recruitment strategies and the intervention by participants and willingness to participate

4. The appropriateness and acceptability of the assessment tools 
5. Preliminary evidence of effects of the intervention on physical activity, mood and anxiousness. This will provide an initial indication of whether the intervention can contribute to change within this group. Estimated effect sizes will provide the parameters for a definitive randomized controlled trial.

\section{Methods And Design}

This protocol conforms to guidelines presented in the Consolidated Standards of Reporting Trials (CONSORT) (33) 2010 statement extension for randomized feasibility studies and clinical trial protocols (see Additional file: Table S1). The intervention component is an uncontrolled open-label feasibility trial (hereafter referred to as 'the intervention'), utilizing a pre-test-post-test within-subject design. The overall study design is illustrated in Fig. 1 (below), whereas the stages of the enrollment, interventions and assessments Table 1 below (The SPIRIT table).

Table 1. SPIRIT table for an uncontrolled open-label feasibility testing of Physical activity intervention: Confident Active and Happy Youth.

\section{Spirit Table}




\begin{tabular}{|c|c|c|c|c|c|}
\hline & \multicolumn{5}{|c|}{ Feasibility test period } \\
\hline \multirow[b]{2}{*}{ TIMEPOINT } & \multirow{2}{*}{$\begin{array}{l}\text { Enrolment } \\
T 1\end{array}$} & \multirow{2}{*}{$\begin{array}{l}\text { Allocation } \\
0\end{array}$} & \multicolumn{2}{|c|}{ Intervention } & \multirow{2}{*}{\begin{tabular}{|l} 
Follow-up \\
T3 \\
(Follow-up)
\end{tabular}} \\
\hline & & & Week 1-7 & $T 2$ & \\
\hline \multicolumn{6}{|l|}{ ENROLMENT: } \\
\hline Eligibility screen & $x$ & & & & \\
\hline Informed consent & $x$ & & & & \\
\hline Baseline assessment & $x$ & & & & \\
\hline Allocation & & $x$ & & & \\
\hline \multicolumn{6}{|l|}{ INTERVENTIONS: } \\
\hline \multicolumn{6}{|l|}{$\mathrm{CAH}-\mathrm{Y}$ Intervention } \\
\hline \multicolumn{6}{|l|}{ ASSESSMENTS: } \\
\hline Actigraph & $x$ & & $x$ & $\mathrm{x}$ & $x$ \\
\hline $\begin{array}{l}\text { Baseline data ( demographic, } \\
\text { psychiatric) }\end{array}$ & $\mathrm{x}$ & & & & \\
\hline Biometric data (height, weight) & $x$ & & & $\mathrm{x}$ & $x$ \\
\hline Feasibility outcomes & $x$ & & $x$ & $x$ & $x$ \\
\hline$S C A S-C / P, S M F Q, N M L-C$ & $x$ & & & $\mathrm{x}$ & $x$ \\
\hline Qualitative interview & $x$ & & & $x$ & $x$ \\
\hline
\end{tabular}

\section{Intervention (CAH-Y)}

The $\mathrm{CAH}-\mathrm{Y}$ intervention includes two sessions of 50 min. per week, for seven weeks, in total 14 sessions. However, the final session is approximately 3 hours long since it includes a nature hike and a conclusion of the program. Several constraints shaped the possible length, intensity and overall dosage of the intervention. This delivery format is a compromise between evidence from behavior change research, organizational considerations and clinical considerations regarding the logistical and practical burden on 
youth and parents (time spent to commute to treatment, time away from school, etc.). Importantly, despite these constraints and consequently the compromises this entails on treatment format, the intended dose of physical activity within $\mathrm{CAH}-\mathrm{Y}$ is still sufficient for the intended mechanisms to have effect (e.g. moderate to vigorous activity, inhibitory learning, building motivation, etc.).

The intervention is group-based with up to eight participants in each group. Two therapists lead each group. Groups are divided into a child group and an adolescent group to accommodate maturational differences. Youth aged 8-13 receive the child intervention, whereas youth aged 14-18 receive the adolescent version. Both versions follow the same format and structure and have the same guiding principles and goals. However, the specific activities in $\mathrm{CAH}-\mathrm{Y} 13$ and $\mathrm{CAH}-\mathrm{Y} 18$ are tailored to the meet the maturational level and interests of the different age groups.

Each session follows the same structure and format (with exception of the last session). This structure consists of, an introduction and warm-up (10 minutes), main activity (35 minutes) and a session wrap-up (5 minutes). The separate sessions have separate overarching topics and include a variety of activities. Sessions take place in a gymhall, two sessions involve activities in a swimming pool and the last session is outside.

Concerning the more specific physical activities in the intervention, sessions include a mix of aerobic (e.g. running, jumping, traversing an obstacle course), resistance (e.g. squats, push-ups) and relaxation exercises (e.g. yoga exercises). The goal for each session is a minimum of $30 \mathrm{~min}$. spent in moderate to vigorous activity.

Specifically during the introduction and warm-up, the therapists give psychoeducation to the youth regarding the bidirectional interplay between thoughts, feelings, actions and particularly the role of avoidance and alternative coping strategies. The exercises are designed to stimulate some distress, fear and/or discomfort, and the therapists couple these feelings and reactions to the introductory psychoeducation, and use the exercises as exposure therapy. A supportive and mastery motivational climate facilitates the individuals need for relatedness (25) and a safe space to practice exposure (being observed), provides an optimal environment to help the youth address and overcome this fear (34). Thus, the group format allows youth to observe other youth, allowing them to observe that other youth have similar feelings, fears, reactions, behavior and responses as themselves, e.g. we sweat when we are physically active, trying out new activities may make us feel anxious but can be done nonetheless. Gaining this knowledge can help normalize the youth's understanding of both themselves and others. Importantly, group and peer support are identified as an important intervention component to support active lifestyles $(35,36)$

In accordance with self-determination theory, the sessions also strive to satisfy the youths' needs for autonomy (e.g., giving them choices within a palette of activities to do), competence (e.g., adapting exercises to meet the needs/fitness levels of students), and relatedness (e.g., focusing on group cohesion, group cooperation). Importantly in this regard, the sessions do not have a competitive focus, but focus on being supportive, enjoyable and fun. An overview of the CAH-Y intervention is presented in Table 2. 
Table 2. Description of the intended structure and format of Confident, Active and Happy Youth program.

\begin{tabular}{|c|c|c|}
\hline $\begin{array}{l}\text { Introduction } \\
\text { Session } 1 \text { - } 2\end{array}$ & $\begin{array}{l}\text { Treatment stage } \\
\text { Session 3-12 }\end{array}$ & $\begin{array}{l}\text { Closing and } \\
\text { transitioning } \\
\text { Session } 13-14\end{array}$ \\
\hline Main Theme: & Main Theme: & Main Theme: \\
\hline Alliance and motivation & Challenge and exposure & $\begin{array}{l}\text { Transitioning to daily } \\
\text { life }\end{array}$ \\
\hline $\begin{array}{l}\text { Feeling secure in the } \\
\text { group }\end{array}$ & & Active "lifestyle" \\
\hline $\begin{array}{l}\text { Alliance to the } \\
\text { group/therapist }\end{array}$ & $\begin{array}{l}\text { From avoidance to exposure Strengthening } \\
\text { motivation }\end{array}$ & $\begin{array}{l}\text { Continue exposure to } \\
\text { PA }\end{array}$ \\
\hline Motivation to partake & & $\begin{array}{l}\text { Motivation to } \\
\text { continue }\end{array}$ \\
\hline Week 1 & Week 2-6 & Week 7 \\
\hline \multirow[t]{2}{*}{2 sessions of $50 \mathrm{~min}}$. & 10 sessions of $50 \mathrm{~min}$. & 1 sessions of $50 \mathrm{~min}$. \\
\hline & 2 sessions weekly & 1 session of 3 hours \\
\hline
\end{tabular}

Notes: Pa: Physical activity.

\section{Therapists}

Group-leaders will have knowledge of mental health disorders, youth development, group-dynamics and physical activity to adequately understand and be able to meet the needs of the youth. Thus, a complementary team consisting of mental health nurse and a youth physiotherapist was recommended by the steering committee, to lead the intervention. Additionally, the need to be able to rapidly address and intervene if youths experience discomfort or need adjustments to activities while still instructing the other youth, mean that at least two therapists are needed.

\section{Study population and eligibility}

Youth are referred to the treatment program from their attending therapist at the local CAMHS. Youth can be included in the study if they meet the criteria below.

Inclusion criteria are:

- Age 8- 17 years

- Symptoms of anxiety and/or depression 
- Youth display reduced daily physical activity (less than 30 mins. pr. day and/or does not partake in physical leisure activities, and/or does not participate in physical education in school).

- The youth is motivated to partake in physical activity

Exclusion criteria are:

- Physical activity is not advised for medical reasons

- Severe learning disabilities and the youth is unable to understand the study protocol

- Specific psychiatric disorders including any eating disorder, psychosis

- Severe challenging behavior or other needs requiring constant one to one support

\section{Recruitment}

Youth will be recruited from CAMHS, Department of Child and Adolescent Psychiatry, Haukeland University Hospital, Norway. CAMHS is organized at the same intervention level as ordinary hospital services (Haukeland University Hospital, Bergen, Norway) and is as such a tertiary specialized service. In the Norwegian context, this is the primary source of treatment for youth with mental health disorders. CAMHS is organized in three inpatient wards, three specialized clinics and seven outpatient clinics. These units together form the recruitment base for $\mathrm{CAH}-\mathrm{Y}$. CAMHS offers all forms of mental health services to youth in a catchment area of app. 460.000 citizens in total. The CAMHS receives app. 3000 referrals per year and delivers treatment to $5.2 \%$ of all youth in the catchment area (37). Taking into account CAH-Y inclusion and exclusion criteria, we estimate that $40 \%$ of these youth qualify for participation in the intervention $(n=600)$. The likely numbers of patients referred and eligible should greatly exceed the numbers required by the feasibility study, meaning well below $10 \%$ of those eligible are required to consent.

Given that $\mathrm{CAH}-\mathrm{Y}$ is organized within CAMHS, this enables easy access to potential participants and streamlined recruitment strategies. In practice, this translates into:

- uncomplicated dispersion of information regarding the intervention to the departments and mental health professionals via intranet, email and/or physical/digital meetings

- ease of implementing an in-house digital referral system

- a low threshold for communication between the $\mathrm{CAH}-\mathrm{Y}$ team and the referring specialist

Based on the therapist's assessment of eligibility and participant's interest in the intervention, the therapist sends a formal referral to the $\mathrm{CAH}-\mathrm{Y}$ team. The referrals are assessed with respect to inclusion/exclusion criteria by the Principle Investigator (PI is the first author). If the youth is assessed eligible, an invitation to attend a recruitment and inclusion interview is sent to the youth (and parents if the youth is below 16 years of age). Enclosed in the letter are also a number of health assessment questionnaires to fill out. The questionnaires assess mood, anxiety, demographic information and motivation to attend the intervention. Finally, this letter informs of a webpage address, where the 
participant can find more information about the intervention and the study. The referring specialist will receive notification that the youth is eligible and is invited to an interview. Approximately a week prior to the interview, youth/parents are contacted via phone and they are given further information about the intervention and study participation.

\section{Consent}

At the recruitment and inclusion interview, participants are provided further information about the intervention and the study, and any ensuing questions can be dealt with. Written information detailing the intervention, the study as well as the consent form are reviewed with the participant and/or parents if they participate in the interview. Informed written consent is obtained from all parents and assent is obtained from youth above age 12 years. All participants in the intervention fill out the same questionnaires, and

go through the same assessment interview. Importantly, participation in the study requires written consent, but participation in the intervention is not dependent on participation in the study.

\section{Withdrawal of the study participants}

At any time, participants can withdraw consent with no consequences for further intervention participation or other eventual benefits. If there are any serious adverse events e.g. injury from the activities, details will be recorded and reported to the PI and to the work management group. CAMHS has appropriate insurance of the participants, if any adverse event should take place. In the case of adverse psychiatric effects are uncovered, this will be reported immediately to the participants referring specialist in CAMHS.

\section{Feasibility procedures}

After referral assessment of eligibility, eligible youth and their primary caregivers are invited to attend a recruitment and inclusion interview. This interview will take place in the CAH-Y facilities. In the interview, therapists will provide further information about the intervention and study details, clarifying any questions and/or concerns, obtaining written consent and they collect and check the questionnaires included in the invitation for the recruitment interview. Moreover, additional questionnaires will be filled out (see primary and secondary outcome measures section), medical information gathered (height and weight), participants will be provided an Actigraph activity monitor to be worn the next seven days and an activity diary to fill out. In addition, a short qualitative interview exploring participant and caregiver views on physical activity will be conducted. Finally, the youth and caregivers will be given a tour of the $\mathrm{CAH}-\mathrm{Y}$ facilities in order to familiarize them with the setting.

The first session of the CAH-Y program will be between one to a maximum of three weeks after the interview. At this point, they will also return the Actigraph monitors and activity diaries. The intervention will then start. The day before every session, the participants will receive a SMS reminder of the session. After each session, participants are encouraged to visit a designated webpage for the program, where they can find further information about the next session. This is in order to remind the participants of the session clarify what is expected and encourage participation. 
Following the last session of the intervention, participants will receive an invitation to attend a postintervention interview, one to a maximum of three weeks following last intervention session. In this interview, questionnaires relating to symptom change will be completed, biometric data will be gathered and a short qualitative interview exploring the views of the participant and his/her caregivers in relation to the intervention will be done. At the end of the interview, participants will be provided an Actigraph activity monitor to be worn for the next seven days and a complementing activity diary. After these seven days have elapsed, a research assistant will gather the Actigraph. Independently of this assessment with the participant, a short qualitative interview with the referring specialist will also be conducted by the PI.

At six months post-intervention, the participants and their caregivers will be invited to attend a follow-up interview. In this interview, questionnaires relating to initial symptoms will be filled out anew, and a new short qualitative interview exploring views possible negative and/or positive effects of the intervention will be done. It the end of the interview, participants will be provided an Actigraph activity monitor to be worn for the next seven days and a complementing activity diary. After this time-period, the Actigraph will gathered by a research assistant. See Fig. 1 for study flow chart.

\section{Comparison group}

Participants will serve as their own controls. Pre- and post-treatment results will be compared.

\section{Measures}

Apart from the measures that participants and their caregivers fill out at home [SCAS, SMFQ, demographics], all other measures and assessments will be conducted in the $\mathrm{CAH}-\mathrm{Y}$ facilities.

\section{Socio-demographic characteristics and psychiatric conditions}

Primary caregivers will provide socio-demographic information. The PI will acquire psychiatric/medical information, via the participant's digital medical records. Axis-1 diagnoses are given during ordinary clinical practice by the youth's psychiatrist or psychologist after reaching a consensus with other professionals from the multi-disciplinary team. In this study, we will classify the patients according to their main Axis I psychiatric diagnoses (ICD-10 codes are specified in Table 2) in the following groups: depressive disorders, anxiety disorders, hyperkinetic disorders, pervasive developmental disorders and other disorders (a broad spectrum of psychiatric disorders with low frequency). The assessment and diagnosis of youth in CAMHS will provide the necessary information in order to asses study eligibility according to the predetermined inclusion/exclusion criteria.

\section{Feasibility outcomes}

Feasibility of the trial will be done by way of continuous tracking of participant recruitment (number of youth referred/number of youth assessed as eligible), attendance and retention. The appropriateness, practicality and acceptability of the intervention within the CAMHS setting, will be explored through feedback from participants, primary caregivers and the referring specialists. This information will be 
gathered via semi-structured qualitative interviews, during the pre- and post-treatment assessment, while the referring specialists will be interviewed separately following the post-treatment assessment. Furthermore, the researchers will meet with the steering group at the end of the study to discuss the results and intervention acceptability.

\section{Participant-centered outcome measures.}

Objective measures of physical activity will be collected using the wearable activity sensor Actigraph GT3X+ monitor. The sensor captures and records continuous, high-resolution physical activity and sleep/wake information and can record daily time spent in sedentary, light and moderate to vigorous physical activity (MVPA). Other research has documented this method to be valid and reliable (38). Predefined minimum wear time for a valid day will be defined as $6 \mathrm{H} /$ day with a minimum of 3 days of data required for analysis inclusion (39). Data for the Actigraph will be downloaded using Actigraph Actilife software and interpreted using 30 second epochs and the following cut-off points: sedentary $(<100 \mathrm{cpm})$, MVPA ( $\geq 3200 \mathrm{cpm})(40)$. Non-wear time will be defined as 60 minutes of consecutive zero counts. Participants will also be asked to wear the Actigraph during each intervention session to provide data on physical activity and MVPA content.

Participant height will be measured in meters and rounded to the nearest $0.1 \mathrm{~cm}$. Weight will be measured in kilograms, and rounded to the nearest $0.1 \mathrm{~kg}$.

Anxiety symptoms will be assessed using Spence Child Anxiety Scale, child and parent version (SCASC/P) (Spence, 1998). The SCAS comprises 38 items rated on a 4-point scale $(0=$ never, $1=$ sometimes, 2 = often, 3 = always), with a maximum score of 114 . SCAS-C/P has demonstrated good psychometric qualities (41).

Affective symptoms will be assessed using Short Mood and Feelings Questionnaire, child and parent version (SMFQ-C/P) (Angold, Costello, Messer, \& Pickles, 1995) is used to assess youth affective symptoms. The SMFQ has demonstrated good psychometric qualities (42).

Intervention motivation will be assessed using Nijmegen Motivation List Child (NML-C) (43) is used to assess treatment motivation in children and adolescents. The NML-C comprises 15 items rated on a 3point scale.

The semi-structured qualitative interview will apart from feasibility outcomes also assess participant perceptions on PA in general, participant views on the association between PA and mental health, perceptions on the intervention components and the intervention as a whole, views on potential barriers to physical activity, motivation for activity and participation in school-based physical activity. These questions are posed at post-treatment and long-term follow-up. Caregivers are posed the same questions; however, caregivers to youth aged 12 years or above, will only receive these questions if the youth has consented to this. Answers to the questions are written down during the interviews and will be analysed 
by thematic analysis (44). An interview with the referring specialist, following the post-treatment interview with the participant, will similarly explore his/her experiences of the intervention including acceptability of procedures, perceived benefits and difficulties.

An effect-size will be calculated for youth and caregiver reported depression and anxiety symptoms and activity level. Means $(M)$ and Standard deviations $(S D)$ will be used to investigate the effect sizes for change between pre- to post-treatment. These results will help inform the power calculation of the likely required sample size for a future large-scale RCT.

\section{Timing}

Measures will be assessed at baseline (pre-intervention interview) and at the latest two weeks after completing the intervention. Follow-up outcome will be assessed six months post-intervention.

\section{Sample size}

As this will be the feasibility study to inform the design of the future definitive RCT, a target sample of 20 youth participants will be recruited for estimation of the intended variables (45).

\section{Progression criteria}

A feasibility studies is not adequately powered to test the any hypothesis (eg. the efficacy of CAH-Y physical activity intervention) but is set to improve the chances of conducting a high-quality RCT. Therefore, in line with suggestions by el-Kotob et al. (46) a priori criteria for progression to the definitive large-scale RCT is advisable to consider future efforts. The progression criteria to move on to a definitive large-scale RCT were A) no serious adverse events, such as hospitalization, a life-threatening condition, death and any adverse events associated with the intervention; B) recruitment rate of no less than $75 \%$; and $C$ ) retention rate of no less than $60 \%$ in each group at 7 weeks (total of 14 sessions). If all the three criteria are not met, we assess there is insufficient evidence to justify proceeding to the definitive RCT. Consequently, changes to the intervention would then be required with consequent re-runs of the intervention following, up until these criteria are met.

\section{Statistics and data analysis}

Quantitative data will be analyzed using SPSS version 22.0 for Windows (SPSS Inc., Chicago, IL).

Baseline data for participants will also be presented in charts as well as any possible participant missing data (questionnaires and Actigraph). Descriptive data will use 95\% confidence intervals (mean and standard deviations). Baseline differences between groups (e.g. age, activity level, BMI, number of mental health disorders and questionnaire outcomes) will be analyzed using one-way ANOVA. Nominal data (e.g. gender, ethnicity, social class, participation in school physical activity) will be analyzed using Chi-square analyses. However, as mentioned the feasibility study is underpowered to detect any effects reliably. Thus, quantitative feasibility outcomes will be interpreted only as feasibility and pilot data due to lack of statistical power. 
Qualitative interviews with participants, caregivers and referring specialists will be analyzed utilizing thematic analysis (44). Accordingly, the written interview data will be reviewed for data familiarization; initial codes will be generated, followed by organizing codes into themes, refining themes, and finally defining themes and sub-themes.

\section{Possible harms}

Potential harms of being involved in the intervention and the assessments will be explicitly outlined in the participant explanatory statements and consent form. During assessment, the primary potential risk for participants may be to experience some psychological distress; however, this is not anticipated to exceed levels of psychological distress experienced previously in CAMHS or in their daily lives. During the intervention, the youth are expected to engage in physical activity with varying degrees of activity intensity. During such activities, the youth could potentially injure themselves or others physically, and/or they could experience some physio-psychological distress. All activities required in the intervention are preplanned and will be completed so as be to be as safe as possible, yet any movement (in a group) does necessarily entail some risk of injury. All accidents and injuries will be recorded and reported to the $\mathrm{PI}$ and the work management group. In addition, any adverse events or mental states observed among the participants (e.g. significant symptom deterioration, suicidal ideation or suicid attempt, reported or observed abuse and/or self-harm, excessive weight loss etc.) will be monitored routinely throughout the study. Any such adverse events will be immediately reported back to the youth's referring specialist, who is responsible for the youth's mental health care. All study therapists and interviewers are experienced in working with youth with anxiety and depression and in responding to distress.

\section{Discussion}

The aim of the current study is to develop and feasibility test a supplemental physical activity program for youth with internalizing disorders in regular treatment in child and adolescent mental health clinics (CAMHS). In light of high prevalence rates of anxiety and depression among children and adolescents, negative short- and long-term consequences, poor recovery rates even when provided best available treatment, there is an immediate need for new and/or supplemental treatment approaches. Physical activity may be one such approach. While the effects of physical activity on internalizing disorders in adults are to some degree empirically underpinned, the benefits and supplemental treatment effects PA may have for youth is only just beginning to evolve. Thus, there exists a clear evidence gap in regards to the effects of PA on youth internalizing disorders and the integration of such an approach in CAMHS. A necessary first step in developing this field and addressing this knowledge gap is developing and feasibility testing a theoretically based physical activity based intervention targeting anxiety and depressive symptoms in youth. This may be an important step towards addressing the burden and consequences these mental health disorders cause youth and to help provide effective treatment.

We anticipate that participation in the intervention will result in increases in physical activity from pre- to post-treatment. Similarly, we also expect some improvements in anxiety and depressive symptomatology from pre- to post-treatment. These anticipated changes are hypothesized to map onto positive changes in 
youth confidence, mood and physical activity. Concerning feasibility testing, we expect that feasibility estimates for recruitment, completion and retention will be met, and results of outcome measures will enable an effect-size estimation for future RCT planning. It is anticipated that youth, parents and referring specialists will find the intervention acceptable. The qualitative component will provide a unique opportunity to gain a rich perspective of the youths and parents experience and acceptability, which is essential towards future planning and trialing of the $\mathrm{CAH}-\mathrm{Y}$ intervention.

\section{Limitations of the research}

As this is a small scale feasibility study with no control group, it is not possible to secure blinding throughout the study. Two therapists provide the recruitment and inclusion assessment interviews and deliver the intervention. However, some of the post-intervention assessments will be done by the study PI as well as the interview with the referring specialist and follow-up interview. Whilst a double-blind feasibility design would strengthen the study, time and resources are limited and do not allow for this. However, in the next steps of the development of the intervention (in line with the MRC model); including a pilot RCT and the definitive RCT, necessary personnel to avoid bias in the trial will be secured.

\section{Strength of the research}

The current study presents a number of strengths. Firstly, a major strength of the study is its alignment with the MRC framework, for the development of complex health interventions. The findings from this study are critical to the next stages of the MRC framework: development and implementation of a definitive RCT exploring the effectiveness of physical activity as a supplemental treatment for youth with internalizing disorders in CAMHS. Secondly, a major strength of the study is a high degree of "real-world" or external validity. Many treatment development studies are based in university settings or specialized clinics, and lead on to efficacy studies. The current study will both develop and aims to test effectiveness in a community setting in the longer-term, thus increasing is applicability in real-life settings and potentially bypassing any limitations and issues in transportability from specialized to community clinic.

Thirdly, the target population for the intervention is a youth population which is especially susceptible to high levels of sedentary time and low levels of physical activity. Thus, this is a vulnerable target population in which changes in physical activity level can have a major impact both short-term and in regards to long-term health outcomes. Given that participants give consent for access to medical journals, this will provide valuable insight into how comorbid illness influence feasibility, but also comorbidity may be affected by the $\mathrm{CAH}-\mathrm{Y}$ intervention.

\section{Trial Status}

The study is currently in progress, and participants are being assessed for eligibility and inclusion into the feasibility trial. Inclusion started in April 2020 and will continue until the target number of participants is included, likely before the end of 2021. Results from the study will be submitted for publication at the end of this year. 


\section{Abbreviations}

ANOVA: Analysis of variance

CAMHS: Child and Adolescent Mental Health Care

CAH-Y: Confident, Active and Happy Youth

HPA: Hypothalamic-pituitary-adrenal axis

MRC: Medical Council Research Framework

MVPA: Moderate to vigorous physical activity

NML-C: Nijmegen Motivation List Child

PI: Principal Investigator

RCT: Randomized controlled trial

SCAS-C/P: Spence Child Anxiety Scale, child and parent version

SMFQ-C/P: Short Mood and Feelings Questionnaire, child and parent version

\section{Declarations}

\section{Ethics approval and consent to participate}

Ethical approval for the study was received from the Regional Committee for Medical and Health Research Ethics, region West, Norway (no. 30912 REK Vest). Informed written consent is obtained from all parents and assent is obtained from youth above age 12 years. Personal confidentiality is guaranteed. None of the assessments are considered to involve any health risk, and we believe most adolescents will benefit from participation in the $\mathrm{CAH}-\mathrm{Y}$ intervention. Those who do not benefit will still have standard care follow-up in CAMHS. The letter of informed consent emphasizes the participants' right to withdraw from the project at any time, with no need for any explanation.

\section{Consent for publication}

Not applicable.

\section{Availability of data and materials}

The treatment manual for $\mathrm{CAH}-\mathrm{Y}$ is available on request (in Norwegian). Data sharing is not applicable to this article as no datasets were generated or analyzed

\section{Competing interests}


All other authors declare no competing interests.

\section{Funding}

This study did not receive any specific grant from funding agencies in the public, commercial, or nonprofit sectors.

\section{Authors' contributions}

PJBT initiated the development of the CAH-Y intervention. AK drafted the trial protocol, and all other authors have contributed to refinement of the study protocol. AK, LPB, PJBT and IE contribute to the implementation of the study. All authors have approved the final manuscript.

\section{Acknowledgements}

We want to acknowledge Martin Håtuft Solberg, Elsa-Kristine Paus Rasch, Per Christian Larsen for their substantial effort in establishing the groundwork for the intervention and implementing the intervention in CAMHS. Also, Elsa-Kristine and Per Christian have played an essential role in the design of the intervention.

\section{References}

1. Polanczyk GV, Salum GA, Sugaya LS, Caye A, Rohde LA. Annual Research Review: A meta-analysis of the worldwide prevalence of mental disorders in children and adolescents. J Child Psychol Psychiatry. 2015;56(3):345-65.

2. Merikangas KR, He JP, Burstein M, Swanson SA, Avenevoli S, Cui L, et al. Lifetime prevalence of mental disorders in U.S. adolescents: results from the National Comorbidity Survey ReplicationAdolescent Supplement (NCS-A). J Am Acad Child Adolesc Psychiatry. 2010;49(10):980-9.

3. WHO. Depression and other common mental disorders: global health estimates. World Health Organization; 2017.

4. Copeland WE, Angold A, Shanahan L, Costello EJ. Longitudinal patterns of anxiety from childhood to adulthood: The Great Smoky Mountains Study. Journal of the American Academy of Child Adolescent Psychiatry. 2014;53(1):21-33.

5. WHO. Adolescent mental health in the European Region. 2018.

6. Wergeland GJH, Riise EN, Öst L-G. Cognitive behavior therapy for internalizing disorders in children and adolescents in routine clinical care: A systematic review and meta-analysis. Clin Psychol Rev. 2021;83:101918.

7. Weisz JR, Kuppens S, Ng MY, Eckshtain D, Ugueto AM, Vaughn-Coaxum R, et al. What five decades of research tells us about the effects of youth psychological therapy: A multilevel meta-analysis and implications for science and practice. Am Psychol. 2017;72(2):79-117. 
8. Ollendick A, Commentary on Cognitive Behavior Therapy. Where We Have Been, Where We Are, and Where We Need to Go From Here. Cogn Behav Pract. 2016;23(4):436-40.

9. Biddle S, Ciaccioni S, Thomas G, Vergeer I. Physical activity and mental health in children and adolescents: An updated review of reviews and an analysis of causality. Psychol Sport Exerc. 2019;42:146-55.

10. Strine TW, Mokdad AH, Dube SR, Balluz LS, Gonzalez O, Berry JT, et al. The association of depression and anxiety with obesity and unhealthy behaviors among community-dwelling. US adults. 2008;30(2):127-37.

11. Firth J, Siddiqi N, Koyanagi A, Siskind D, Rosenbaum S, Galletly C, et al. The Lancet Psychiatry Commission: a blueprint for protecting physical health in people with mental illness. The Lancet Psychiatry. 2019;6(8):675-712.

12. Rebar AL, Stanton R, Geard D, Short C, Duncan MJ, Vandelanotte C. A meta-meta-analysis of the effect of physical activity on depression and anxiety in non-clinical adult populations. Health Psychology Review. 2015;9(3):366-78.

13. McDowell CP, Dishman RK, Gordon BR, Herring MP. Physical Activity and Anxiety: A Systematic Review and Meta-analysis of Prospective Cohort Studies. Am J Prev Med. 2019;57(4):545-56.

14. Stubbs B, Vancampfort D, Rosenbaum S, Firth J, Cosco T, Veronese N, et al. An examination of the anxiolytic effects of exercise for people with anxiety and stress-related disorders: A meta-analysis. Psychiatry Res. 2017;249:102-8.

15. Stubbs B, Vancampfort D, Hallgren M, Firth J, Veronese N, Solmi M, et al. EPA guidance on physical activity as a treatment for severe mental illness: a meta-review of the evidence and Position Statement from the European Psychiatric Association (EPA), supported by the International Organization of Physical Therapists in Mental Health (IOPTMH). Eur Psychiat. 2018;54:124-44.

16. Wegner M, Amatriain-Fernández S, Kaulitzky A, Murillo-Rodriguez E, Machado S, Budde H. Systematic Review of Meta-Analyses: Exercise Effects on Depression in Children and Adolescents. 2020;11(81).

17. Bailey AP, Hetrick SE, Rosenbaum S, Purcell R, Parker AG. Treating depression with physical activity in adolescents and young adults: a systematic review and meta-analysis of randomised controlled trials. Psychol Med. 2018;48(7):1068-83.

18. Axelsdóttir B, Biedilæ S, Sagatun Å, Nordheim LV, Larun L. Exercise for depression in children and adolescents-a systematic review and meta-analysis. Journal of Child Adolescent Mental Health. 2020.

19. Carter T, Pascoe M, Bastounis A, Morres ID, Callaghan P, Parker AG. The effect of physical activity on anxiety in children and young people: a systematic review and meta-analysis. J Affect Disord. 2021;285:10-21.

20. Lubans D, Richards J, Hillman C, Faulkner G, Beauchamp M, Nilsson M, et al. Physical Activity for Cognitive and Mental Health in Youth: A Systematic Review of Mechanisms. Pediatrics. 2016;138(3):e20161642. 
21. Schuch FB, Deslandes AC, Stubbs B, Gosmann NP, Silva CTBd, Fleck MPdA. Neurobiological effects of exercise on major depressive disorder: A systematic review. Neuroscience Biobehavioral Reviews. 2016;61:1-11.

22. Wegner M, Helmich I, Machado S, Nardi E, Arias-Carrion A, Budde O H. Effects of Exercise on Anxiety and Depression Disorders: Review of Meta- Analyses and Neurobiological Mechanisms. CNS \& Neurological Disorders - Drug Targets- CNS \& Neurological Disorders). 2014;13(6):1002-14.

23. Hillman PM, Castelli B, Khan DM, Raine NA, Scudder LB. M, R., et al. Effects of the FITKids Randomized Controlled Trial on Executive Control and Brain Function. Pediatrics. 2014;134(4):e1063e71.

24. Kertz SJ, Belden AC, Tillman R, Luby J. Cognitive Control Deficits in Shifting and Inhibition in Preschool Age Children are Associated with Increased Depression and Anxiety Over 7.5 Years of Development. J Abnorm Child Psych. 2016;44(6):1185-96.

25. Ryan RM, Deci EL. Self-determination theory and the facilitation of intrinsic motivation, social development, and well-being. Am Psychol. 2000;55(1):68-78.

26. Silverman WK, Field AP. Anxiety disorders in children and adolescents. Cambridge University Press; 2011.

27. APA. Diagnostic and statistical manual of mental disorders, (DSM-5®). American Psychiatric Pub; 2013.

28. Mangerud WL, Bjerkeset O, Lydersen S, Indredavik MS. Physical activity in adolescents with psychiatric disorders and in the general population. Child Adolescent Psychiatry Mental Health. 2014;8(1):2.

29. Kandola A, Vancampfort D, Herring M, Rebar A, Hallgren M, Firth J, et al. Moving to Beat Anxiety: Epidemiology and Therapeutic Issues with Physical Activity for Anxiety. Current Psychiatry Reports. 2018;20(8):63.

30. Craske MG, Treanor M, Conway CC, Zbozinek T. Vervliet BJBr, therapy. Maximizing exposure therapy: An inhibitory learning approach. 2014;58:10-23.

31. Craig P, Dieppe P, Macintyre S, Michie S, Nazareth I, Petticrew MJB. Developing and evaluating complex interventions: the new Medical Research Council guidance. 2008;337.

32. O'Cathain A, Hoddinott P, Lewin S, Thomas KJ, Young B, Adamson J, et al. Maximising the impact of qualitative research in feasibility studies for randomised controlled trials: guidance for researchers. Pilot Feasibility Studies. 2015;1(1):32.

33. Eldridge SM, Chan CL, Campbell MJ, Bond CM, Hopewell S, Thabane L, et al. CONSORT 2010 statement: extension to randomised pilot and feasibility trials. 2016;355:i5239.

34. Diamond A, Ling DS. Conclusions about interventions, programs, and approaches for improving executive functions that appear justified and those that, despite much hype, do not. Dev Cogn Neurosci. 2016;18:34-48.

35. Mitchell F, Gray S, Inchley J. 'This choice thing really works ... Changes in experiences and engagement of adolescent girls in physical education classes, during a school-based physical 
activity programme. Physical Education Sport Pedagogy. 2015;20(6):593-611.

36. Martins J, Marques A, Sarmento H, Carreiro da Costa F. Adolescents' perspectives on the barriers and facilitators of physical activity: a systematic review of qualitative studies. Health Educ Res. 2015;30(5):742-55.

37. Krogh F, Indergård P. Aktivitetsdata for psykisk helsevern for barn og unge 2019.: Oslo: Helsedirektoratet.; 2020.

38. Aadland E, Ylvisåker E. Reliability of the Actigraph GT3X+ Accelerometer in Adults under Free-Living Conditions. PloS one. 2015;10(8):e0134606-e.

39. Basterfield L, Adamson AJ, Pearce MS, Reilly JJJJoPA. Health. Stability of habitual physical activity and sedentary behavior monitoring by accelerometry in 6-to 8-year-olds. 2011;8(4):543-7.

40. Puyau MR, Adolph AL, Vohra FA, Butte NF. Validation and Calibration of Physical Activity Monitors in Children. Obes Res. 2002;10(3):150-7.

41. Spence SH, Barrett PM, Turner CM. Psychometric properties of the Spence Children's Anxiety Scale with young adolescents. J Anxiety Disord. 2003;17(6):605-25.

42. Costello EJ, Angold A. Scales to Assess Child and Adolescent Depression: Checklists, Screens, and Nets. Journal of the American Academy of Child Adolescent Psychiatry. 1988;27(6):726-37.

43. Ollendick TH, Öst L-G, Reuterskiöld L, Costa N, Cederlund R, Sirbu C, et al. One-session treatment of specific phobias in youth: a randomized clinical trial in the United States and Sweden. J Consult Clin Psych. 2009;77(3):504.

44. Braun V, Clarke V. Using thematic analysis in psychology. Qualitative Research in Psychology. 2006;3(2):77-101.

45. Arain M, Campbell MJ, Cooper CL, Lancaster GA. What is a pilot or feasibility study? A review of current practice and editorial policy. BMC Med Res Methodol. 2010;10(1):67.

46. El-Kotob R, Giangregorio LM. Pilot and feasibility studies in exercise, physical activity, or rehabilitation research. Pilot Feasibility Studies. 2018;4(1):137.

\section{Figures}




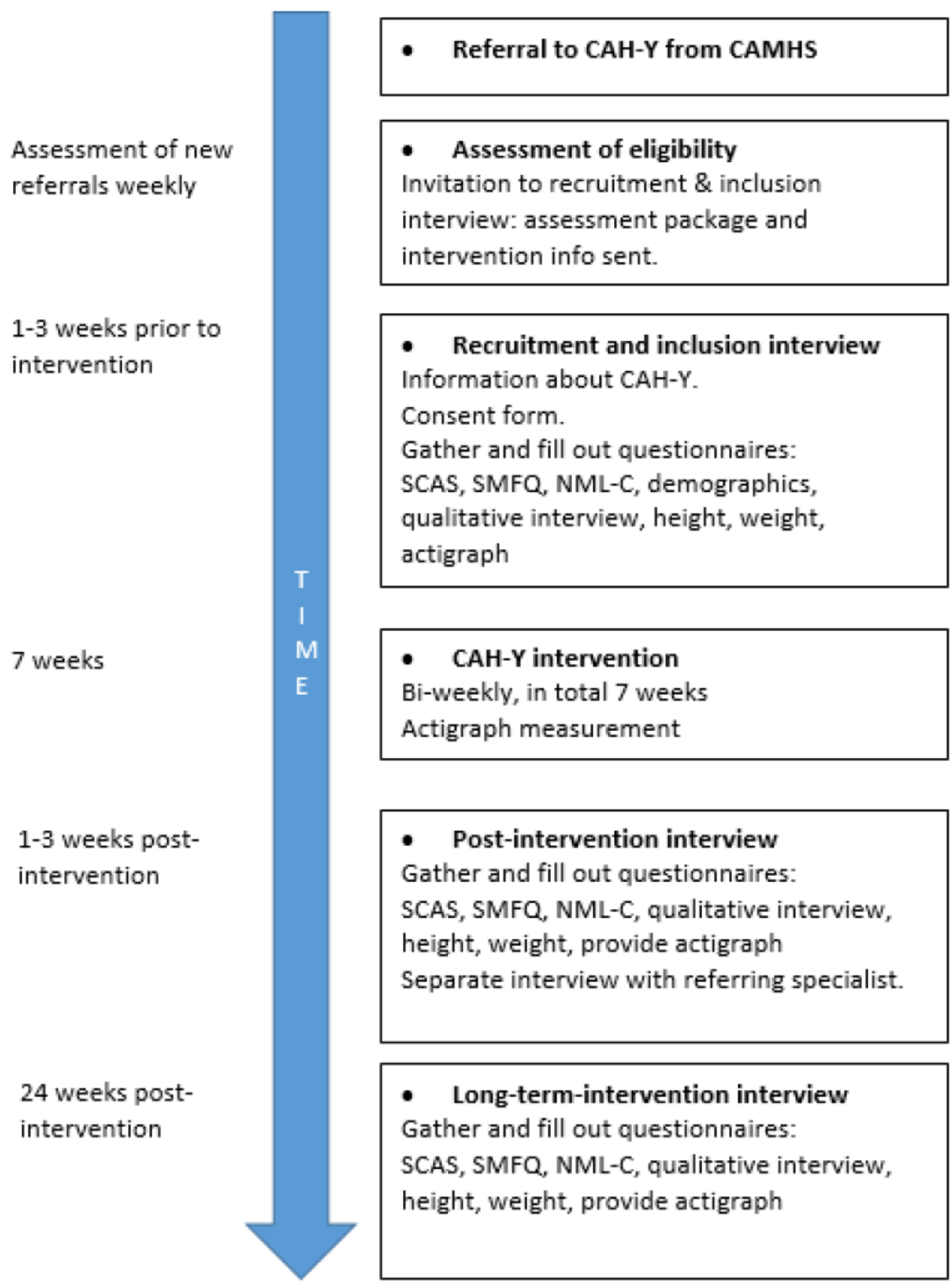

Figure 1

Study flow chart for $\mathrm{CAH}-\mathrm{Y}$ feasibility study.

\section{Supplementary Files}

This is a list of supplementary files associated with this preprint. Click to download. 
- AdditionalFiles.docx

Page 23/23 